\title{
CYP4F2 and CYP3A5 gene polymorphisms and lung cancer in Chinese Han population
}

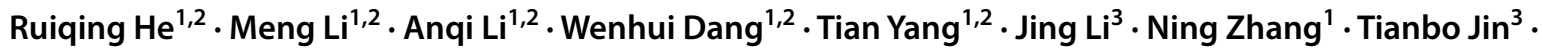 \\ Mingwei Chen ${ }^{1,2}$ (D)
}

Received: 20 March 2020 / Accepted: 18 April 2020 / Published online: 30 April 2020

(c) The Author(s) 2020

\begin{abstract}
This study aimed to explore whether the polymorphisms of CYP4F2 and CYP3A5 are correlated with the risk of lung cancer development. A case-control study was conducted among 510 patients with pathologically confirmed lung cancer as the case group and 504 healthy individuals as the control group. Four single-nucleotide polymorphisms of the CYP4F2 and $C Y P 3 A 5$ genes were genotyped, and their correlations with the risk of lung cancer were examined using Chi-square test and logistic regression analysis. Stratified analysis found that the rs3093105 and rs3093106 loci of CYP4F2 gene were significantly associated with lower risk of lung cancer $(P=0.012$, OR $0.64,95 \%$ CI $0.45-0.91)$. The correlation was related to patients' age and sex and pathological type of lung cancer. Similarly, the rs10242455 loci of CYP3A5 gene showed a statistical significance between the case group and the control group $(P=0.018$, OR $0.71,95 \%$ CI $0.53-0.94)$, which also was associated with reduced risk of squamous cell lung cancer in the dominant and additive models (dominant: OR 0.66, 95\% CI 0.46-0.94, $P=0.021$; additive: OR 0.71, 95\% CI 0.53-0.95, $P=0.023$ ). CYP4F2 and CYP $3 A 5$ gene polymorphisms are associated with the reduced risk of non-small cell lung cancer, and its correlation is related to patients' age and sex and pathological type of lung cancer.
\end{abstract}

Keywords Gene polymorphisms $\cdot$ CYP3A5 $\cdot$ CYP4F2 Lung cancer

\section{Introduction}

Lung cancer is one of the most common malignant tumors [1]. With the increase in detection rate of lung cancer and the aggravation of environmental pollution, morbidity and mortality of lung cancer are increasing year by year [2]. At present, lung cancer has become the leading cause of cancer death worldwide in both sexes combined $(18.4 \%$ of the total cancer deaths) $[3,4]$. According to statistics, in 2012, 1.8

Mingwei Chen

chenmw36@163.com

1 The Department of Respiratory and Critical Care Medicine of the First Affiliated Hospital of Xi' an Jiaotong University, 277 West Yanta Road, Xi'an 710061, Shaanxi, China

2 Shaanxi Provincial Research Center for the Project of Prevention and Treatment of Respiratory Diseases, 1\# Xin Wang Road, Xi' an 710021, Shaanxi, China

3 Ministry of Education Key Laboratory of Resource Biology and Biotechnology in Western China (Northwest University), \#229 North Taibai Road, Xi'an 710069, Shaanxi, China million new cases of lung cancer occurred, accounting for about $13 \%$ of new cancer $[1,4,5]$. Epidemiological studies have demonstrated that lung cancer diagnoses are associated with smoking in highly developed countries [6], but only $10-15 \%$ of smokers develop lung cancer in all smokers, and the morbidity of lung cancer varies in different genders, races, and regions. Studies show that lung cancer has the highest morbidity in North America, East Asia, the Middle East, and the Southern Europe, while female lung cancer rates are highest in North America and Southern Europe [5, 7]. In addition, many studies have shown that the development, metastasis, and prognosis of lung cancer are related to many gene mutations, such as EGFR, KRAS, and BRAF $[8,9]$. The above researches indicated that lung cancer is not only caused by environmental factors, genetic factors cannot be ignored in the occurrence and development of lung cancer.

Cytochrome P450 (CYP450), belonging to $\omega$-hydroxylase, participates in the metabolism of many endogenous substances and exogenous compounds, including fatty acids, docosahexenoic acid, and vitamin $\mathrm{D}$, and of a wide variety 
of carcinogens and anticancer drugs [10, 11]. These reactive metabolites would interact with DNA, thereby causing altered gene expression or function, and eventually carcinogenesis [12]. Therefore, CYP450 may influence tumor genesis and progression. $C Y P 4 F 2$ and $C Y P 3 A 5$ are members of the CYP450 family. A recent study shows that the expression of $C Y P 4 F 2$ is closely related to hepatocellular carcinoma cells, which may contribute to tumor progression [10]. Relative studies demonstrate that 20-HETE (CYP4F2related products) was associated with the growth of tumors in mouse non-small cell lung cancer cell lines [13]. Another study confirmed that CYP3A5 was associated with lung cancer in the population of Taiwan, China [14]. However, little research has been done about the association between CYP4F2 and CYP3A5 gene polymorphisms and lung cancer in the Chinese Han population of mainland China.

Therefore, in this study, four SNP locus in CYP4F2 and CYP3A5 genes were analyzed to explore the association between the polymorphisms of CYP4F2 and CYP $3 A 5$ genes and the risk for lung cancer.

\section{Materials and methods}

\section{Subject recruitment and sample collection}

A case-control study, involving 510 lung cancer patients as the case group and 504 healthy individuals as the control group, was conducted at the First Affiliated Hospital of Xi' an Jiaotong University, Shaanxi, China. All included patients had recently been diagnosed and pathologically confirmed primary lung cancer. The subjects in the control group were recruited from the Health Checkup Center of the First Affiliated Hospital of Xi' an Jiaotong University, who take health examination annually and have no histories of cancers and no chronic or serious endocrine or metabolic nutritional diseases. Patients were ascertained to be free from any acute or chronic pathology. Blood samples from the patients with lung cancer were collected before initiation of chemotherapy or radiotherapy. All of the participants were genetically unrelated ethnic Han Chinese and agree to participate in the present study. The protocols for this study were approved by the Ethical Committees of both the First Affiliated Hospital of Xi' an Jiaotong University and Northwest University.

Five milliliters of whole blood was collected from each subject into tubes containing ethylenediaminetetraacetic acid at the time of initial diagnosis. After centrifugation, the samples were stored at $-80^{\circ} \mathrm{C}$ until further use. The characteristics of all study participants are summarized in Table 1.
Table 1 Characteristics of case group and control group in the study

\begin{tabular}{llll}
\hline Variable & Case $(n=510)$ & Control $(n=504)$ & $P$ value \\
\hline Sex & & & 0.911 \\
Female & $126(24.7)$ & $123(24.4)$ & \\
Male & $384(75.3)$ & $381(75.6)$ & \\
Age, years & $58.08 \pm 10.548$ & $57.27 \pm 10.852$ & 0.227 \\
$\quad$ (mean \pm SD) & & & \\
TNM stage & & & \\
I-II & $129(25.3)$ & & \\
III-IV & $248(48.6)$ & & \\
Miss & $133(26.1)$ & & \\
Pathological types & & \\
SCC & $169(33.1)$ & & \\
AC & $161(31.6)$ & & \\
SCLC & $97(19.0)$ & \\
Others & $22(4.3)$ & & \\
Miss & $61(12.0)$ & \\
\hline
\end{tabular}

SCLC small cell lung cancer

\section{SNPs selection and primer design}

Based on GWAS studies of tumors and reports in related literature, four SNP loci of $C Y P 4 F 2$ and $C Y P 3 A 5$ genes were selected. All loci met the criterion that the minimum allele frequency was more than 5\% in HapMap Chinese Han Beijing population. Primers were designed according to ASSAY Design SUITE V2.0 (https://agenacx.com/online-tools). (All primers were designed according to the sequence of forward strand from dbSNP Database.)

\section{DNA purity detection and genotyping}

DNA was extracted by whole blood genome DNA purification kit (Xi' an GoldMag Biological Company). The concentration and purity of DNA were detected by Nanodrop Lite ultraviolet spectrophotometer (Thermo Scientific, Waltham, Massachusetts, USA). Genotyping of all SNPs was performed on Mass ARRAY iPLEX (Agena Bioscience, San Diego, CA, USA) platform using matrix-assisted laser desorption ionization time of flight (MALDI-TOF) mass spectrometer. The results were output by Agena Bioscience TYPER 4.0 software.

\section{Statistical analysis}

Microsoft Excel (Microsoft, Redmond, WA) and SPSS software(version 19.0, SPSS, Chicago, IL) were used for statistical analysis. Chi-square test was taken to compare the distribution of frequency of suspicious confounding factors 
(age, sex, etc.) in cases and control groups, to determine the comparability between the two groups. Hardy-Weinberg equilibrium test (HWE) was performed on all SNP frequencies in the control group by Chi-square test. Fisher's exact test was used to compare the allele and genotype frequencies of each locus in two groups. We used logistic regression analysis to assess the association between each SNP and the risk of lung cancer and risk for lung cancer in different genetic models (additive, dominant, recessive models), while conducting management considering age and gender. Logistic regression analysis was also used to calculate odds ratios (ORs) and 95\% confidence intervals (CIs). In the comparisons above, a two-sided $P$ value $<0.05$ was considered statistically significant. According to the stratification of age, gender, and pathological types of lung cancer, the correlation between SNP sites and lung cancer risk in different stratified populations was evaluated. The specific method was the same as the above.

\section{Results}

\section{Population characteristics}

510 cases of lung cancer were included in this study. The average age was $58.08( \pm 10.548)$ years old in the cases, of which $75.3 \%$ were males and $24.7 \%$ females. 504 cases were included in the control group, with an average age of 57.27 ( \pm 10.852 ) years old, of which $75.6 \%$ were males and $24.4 \%$ females. Chi-square test showed that there was no significant difference in age and sex between the case group and the control group (age: $P=0.227$, gender: $P=0.911$ ) (Table 1).

\section{SNP and the risk of lung cancer}

Basic information of four SNPs loci in $C Y P 4 F 2$ and $C Y P 3 A 5$ genes is shown in (Table 2). All SNPs loci were in accordance with Hardy-Weinberg equilibrium (HWE) assessed by Chi-square test and Fisher's exact test of SPSS software. The distributions of allele frequencies between the case group and the control group were compared by Chi-square test of Plink software. The results showed that the $P$ values of all SNPs loci in the whole population were greater than 0.05 in allele model, which demonstrated that there was no significant difference between the two groups in the whole population.

From the analysis above, it can be concluded that four SNPs loci in $C Y P 4 F 2$ and $C Y P 3 A 5$ genes have no significant correlation with risk for lung cancer in the case group and the control group in allele model, so stratified analysis was carried out in the aspect of age, sex, and pathological type of lung cancer. Stratified analysis found that three of the four selected SNP loci were significantly associated with lowered risk of lung cancer in allele model, namely rs3093106 (CYP4F2), rs3093105 (CYP4F2), and rs10242455 (CYP3A5), among which rs3093106 and rs3093105 were significantly different between the two groups in the subjects of older than 58 years old (rs3093105: $P=0.023$, OR 0.59, 95\% CI 0.37-0.93; rs3093106: $P=0.029$, OR 0.60, 95\% CI 0.38-0.94), lung adenocarcinoma (rs3093105, $P=0.023$, OR 0.59, 95\% CI 0.37-0.93; rs3093106: $P=0.025$; OR 0.60, 95\% CI 0.38-0.94) and male patients (rs3093105, $P=0.017$, OR 0.68, 95\% CI 0.49-0.93; rs3093106: $P=0.020$, OR 0.68, 95\% CI 0.50-0.94). Rs 10242455 in CYP3A5 gene showed significant difference between the two groups in lung squamous cell carcinoma (rs 10242455: $P=0.018$, OR 0.71, 95\% CI 0.53-0.94) (Tables 3, 4).

In addition, three selected SNPs loci were analyzed in different populations and different genetic models through logistic regression analysis. The results showed that the rs3093105 and rs3093106 were linked with reduced risk of lung cancer in the dominant model and additive model of lung adenocarcinoma, male patients and patients older than 58 years old. After adjusting for age and gender, the correlation was still observed (Table 3 ). The rs10242455 was associated with lowered risk of lung squamous cell carcinoma in the dominant model and additive model; after adjusting for age and gender, the correlation was still observed (Table 4).

In addition, through the analysis of TCGA database, GEPIA database (http://gepia.cancer-pku.cn/), Kaplan-Meier plotter database (http://kmplot.com/), the correlation between expression and prognosis was analyzed.

Table 2 SNP in CYP4F2 and CYP3A5 gene

\begin{tabular}{lllllllll}
\hline SNP ID & Genes & Band & Position & Role & Alleles A/B & $p$-HWE & $P^{\mathrm{a}}$ & OR (95\% CI) \\
\hline rs10242455 & CYP3A5 & 7 & 99642556 & Intron variant & G/A & 0.396 & 0.403 & $0.92(0.76-1.12)$ \\
rs2108622 & CYP4F2 & 19 & 15879621 & Missense & T/C & 0.664 & 0.349 & $0.91(0.75-1.11)$ \\
rs3093106 & CYP4F2 & 19 & 15897447 & Synonymous codon & C/T & 1.000 & 0.128 & $0.80(0.61-1.07)$ \\
rs3093105 & CYP4F2 & 19 & 15897578 & Missense & C/A & 1.000 & 0.112 & $0.80(0.60-1.05)$ \\
\hline
\end{tabular}

SNP single-nucleotide polymorphism, $O R$ odds ratio, 95\% CI 95\% confidence interval, $H W E$ Hardy-Weinberg equilibrium

${ }^{\text {a }}$ Two-sided Chi-square tests; $* p \leq 0.05$ indicates statistical significance 


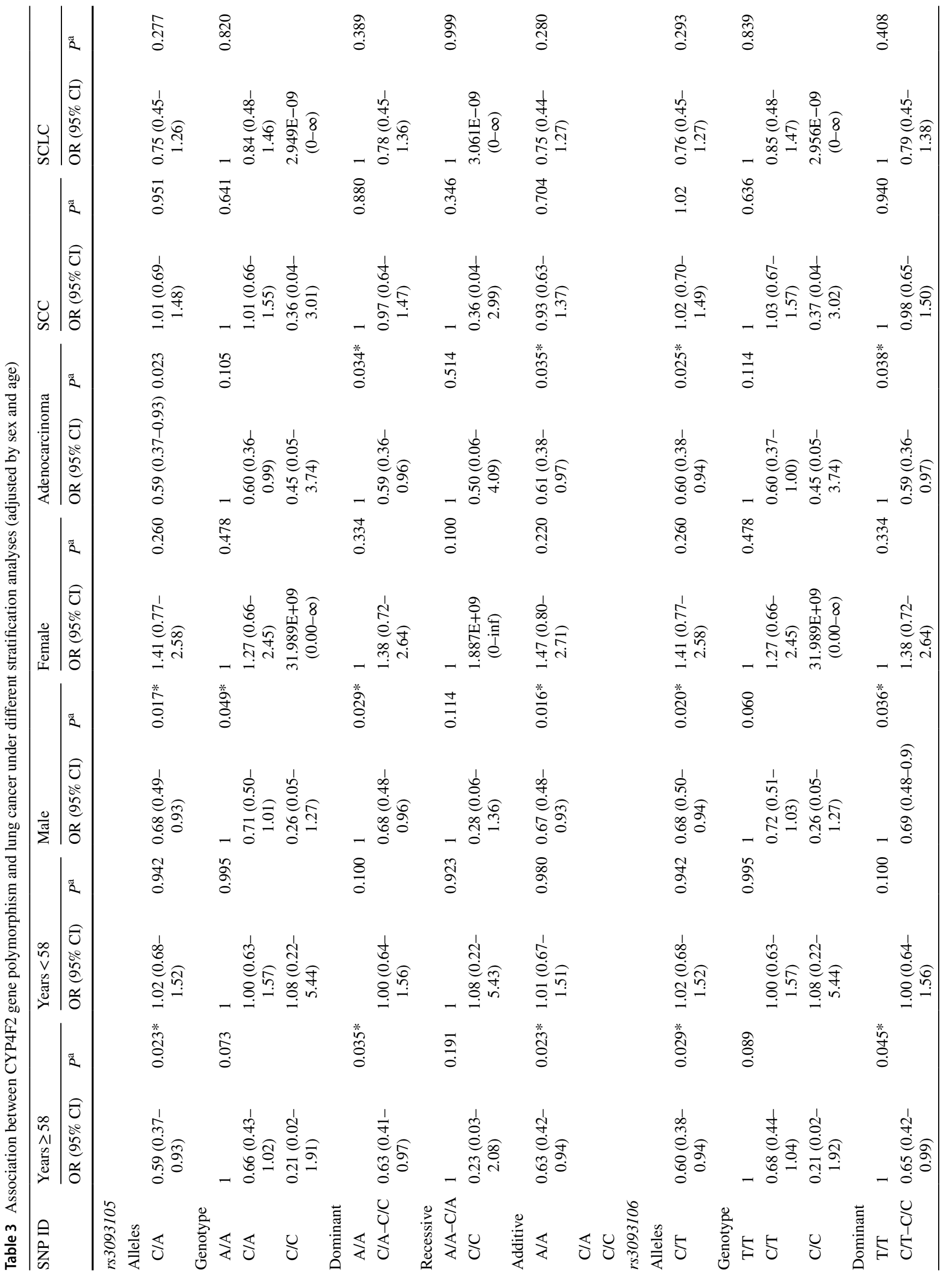


It was found that the expression of CYP3A5 gene in cancer tissues is lower than that in para-tumor tissues (Fig. 1), and there was a worse prognosis in lung cancer patients with lower expression (Fig. 2).

\section{Discussion}

This study suggests that $C Y P 3 A 5$ and $C Y P 4 F 2$ were associated with reduced risk of NSCLC. This was related to age, sex, and pathological type of lung cancer.

$C Y P 4 F 2$ is a member of the CYP $4 F$ family. Several studies have revealed marked mRNA up-regulation of genes encoding CYP4 enzymes in thyroid, breast, colon, and ovarian cancers. Alexanian et al. [15] confirmed that the levels of CYP4F2 and 20-HETE in ovarian cancer tissues were higher than those in normal control group. However, up to now, the correlation between $C Y P 4 F$ gene and lung cancer has not been reported. Our study has been the first to report that there is a significant correlation between $C Y P 4 F 2$ gene polymorphisms and lung cancer in Chinese Han population, and this is associated with lowered risk of lung cancer in people older than 58 years old, lung adenocarcinoma and men. Similarly, Ankit et al. confirmed that the expression of $C Y P 4 F 2$ was increased in pancreatic ductal carcinoma, and the expression of $C Y P 4 F 2$ was negatively correlated with age and higher in males [16]. This is similar to the conclusion of the present study. In addition, many studies have confirmed that $C Y P 4 F 2$ was closely related to the metabolism of 20-hydroxyethyl hexadecanoic acid (20-HETE) [17-19]. In the past decade, 20-HETE has been recognized as a key conditioning agent of cancer progression, which can induce cell proliferation in vitro by stimulating the formation of reactive oxygen species and the production of vascular endothelial growth factor. Previous studies have shown that 20-HETE antagonists (WIT002) can inhibit the proliferation of renal adenocarcinoma [20]. Similarly, two studies have demonstrated that HET0016 (20-HETE antagonist) can inhibit the growth of tumors in non-small cell lung cancer cell lines and of human glioma [13, 21]. We hypothesize that the effect of $C Y P 4 F 2$ gene polymorphisms on the risk for lung cancer may be related to the metabolism of 20-HETE and then affect the growth of cancer cells by regulating the signal pathway of vascular endothelial growth factor. However, further experiments are needed to confirm this.

CYP3A5 is an important member of the CYP3A family. It participates in the catalytic oxidation of many exogenous substances, including toxins, carcinogens, the metabolism, and clearance of some drugs [1]. Studies have shown that CYP3A5 plays an important role in the development of acute and chronic leukemia, colorectal cancer, and esophageal cancer [22-25]. Islam et al. [26] reported that CYP3A5 
Table 4 Association between CYP3A5 gene polymorphism and lung cancer under different pathological types (adjusted by sex and age)

\begin{tabular}{|c|c|c|c|c|c|c|}
\hline \multirow[t]{2}{*}{ SNP ID } & \multicolumn{2}{|l|}{ SCC } & \multicolumn{2}{|l|}{ Adenocarcinoma } & \multicolumn{2}{|l|}{ SCLC } \\
\hline & $\overline{\mathrm{OR}}(95 \% \mathrm{CI})$ & $P^{\mathrm{a}}$ & $\overline{\mathrm{OR}(95 \% \mathrm{CI})}$ & $P^{\mathrm{a}}$ & OR $(95 \% \mathrm{CI})$ & $P^{\mathrm{a}}$ \\
\hline \multicolumn{7}{|l|}{$r s 10242455$} \\
\hline \multicolumn{7}{|l|}{ Alleles } \\
\hline G/A & $0.71(0.53-0.94)$ & $0.018^{*}$ & $1.26(0.96-1.64)$ & 0.091 & $0.82(0.58-1.16)$ & 0.261 \\
\hline \multicolumn{7}{|l|}{ Genotype } \\
\hline $\mathrm{A} / \mathrm{A}$ & 1 & 0.064 & 1 & 0.252 & 1 & 0.317 \\
\hline G/A & $0.67(0.46-0.98)$ & & $1.29(0.88-1.88)$ & & $0.70(0.44-1.11)$ & \\
\hline $\mathrm{G} / \mathrm{G}$ & $0.57(0.27-1.19)$ & & $1.58(0.83-3.02)$ & & $0.85(0.37-1.91)$ & \\
\hline \multicolumn{7}{|l|}{ Dominant } \\
\hline $\mathrm{A} / \mathrm{A}$ & 1 & $0.021 *$ & 1 & 0.126 & 1 & 0.145 \\
\hline $\mathrm{G} / \mathrm{A}-\mathrm{G} / \mathrm{G}$ & $0.66(0.46-0.94)$ & & $1.33(0.92-1.92)$ & & $0.72(0.46-1.12)$ & \\
\hline \multicolumn{7}{|l|}{ Recessive } \\
\hline $\mathrm{A} / \mathrm{A}-\mathrm{G} / \mathrm{A}$ & 1 & 0.274 & 1 & 0.290 & 1 & 0.970 \\
\hline $\mathrm{G} / \mathrm{G}$ & $0.67(0.32-1.38)$ & & $1.40(0.75-2.58)$ & & $0.98(0.44-2.18)$ & \\
\hline \multicolumn{7}{|l|}{ Additive } \\
\hline $\mathrm{A} / \mathrm{A}$ & $0.71(0.53-0.95)$ & $0.023^{*}$ & $1.27(0.96-1.68)$ & 0.097 & $0.81(0.57-1.16)$ & 0.248 \\
\hline \multicolumn{7}{|l|}{$\mathrm{G} / \mathrm{A}$} \\
\hline $\mathrm{G} / \mathrm{G}$ & & & & & & \\
\hline
\end{tabular}

SNP single-nucleotide polymorphism, OR odds ratio, 95\% CI 95\% confidence interval, SCC squamous cell carcinoma, $S C L C$ small cell lung cancer

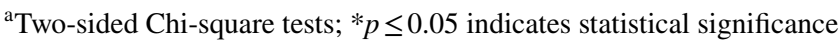

was a risk factor of lung cancer in Bangladeshi population. Interestingly, we found that $C Y P 3 A 5$ was a protective factor of NSCLC in Chinese Han population, which may be related to racial differences. Similarly, in a study of Taiwanese of China, CYP3A5 has been confirmed to play a protective role in the development of lung cancer [14]. Also, Feng et al. indicated that $C Y P 3 A 5$ plays a protective role in the occurrence and metastasis of hepatocellular carcinoma. At the same time, they also confirmed that CYP3A5 overexpression in hepatocellular carcinoma cells inhibits the metastasis and invasion of cancer cells in vivo and in vitro, via manipulating ROS/mTORC2/p-AKT (S473) signaling pathway and limiting MMP2/9 function [25, 27]. Research has found that a SNP within intron-3 (CYP3A5*3) results in aberrant mRNA splicing and a pronounced reduction in protein synthesis [28]. Likewise, rs 10242455 belongs to intron variants in CYP3A5 gene. So, we suspect that CYP3A5 may affect ROS/mTORC2/p-AKT (S473) signaling pathway and limiting MMP2/9 function by affecting mRNA splicing and protein synthesis, thereby affecting the occurrence of lung cancer.
In addition, we found that $C Y P 3 A 5$ gene was low expressed in lung squamous cell carcinomas, and the survival rate was lower among the lung cancer patients with low expression. Similarly, Tingdong suggests that the lower the expression of $C Y P 3 A 5$, the worse the prognosis in hepatocellular carcinoma patients [25]. Another study in Chinese population showed that $C Y P 3 A 5$ gene is closely related to the prognosis of patients with non-small cell lung cancer undergoing chemotherapy and surgical treatment [1]. This is similar to our conclusion. Besides, two recent studies indicated that $C Y P 3 A 5$ gene participates in the metabolism of docetaxel and sunitinib. Different genotypes respond differently to drug dosage requirements and drug toxicity [29, 30]. This suggests that $C Y P 3 A 5$ gene may be related not only to the risk and prognosis of lung cancer, but also to the treatment and drug selection of lung cancer. It may be a predictor of the occurrence, development, and prognosis of lung cancer, but it needs a larger sample of research to further confirm the findings.

Our research confirms that $C Y P 4 F 2$ and $C Y P 3 A 5$ gene polymorphisms are associated with the risk of lung cancer. 


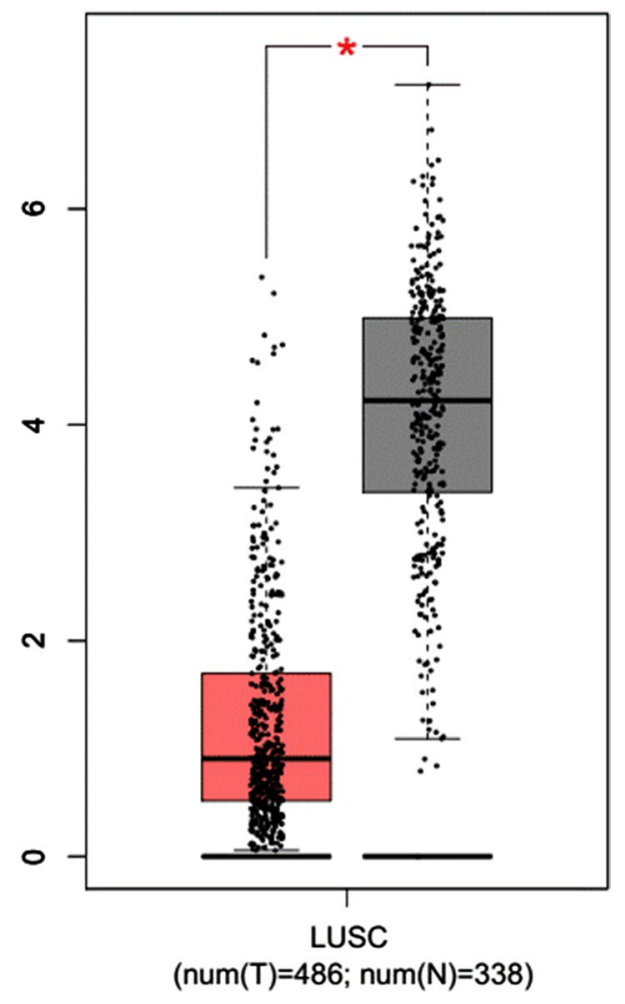

Fig. 1 Expression of CYP3A5 gene in lung squamous cell carcinoma $(n=486)$ and para-tumor tissues $(n=338)$ from GEPIA database. The $Y$-axis is the $\log$-scale of $\log _{2}(\mathrm{TPM}+1)(T P M$ Transcripts Per Million). The box plots show the interquartile range (IQR), median (bar in box), tissues. CYP3A5 expression is significantly lower in lung squamous cell carcinoma $(* p<0.01)$

We believe that our results will encourage more people using larger sample sizes to further confirm the relationship between $C Y P 4 F 2$ and $C Y P 3 A 5$ genes and lung cancer, as well as their specific mechanisms in the occurrence and development of lung cancer in the future studies. But there are still limitations for our study. First, the treatment and survival time of lung cancer patients did not take into consideration. Second, we did not collect the smoking data of the samples in our study, and further study is needed to improve the deficiencies of this research.

\section{Conclusion}

This study found that CYP4F2 and CYP3A5 gene polymorphisms were associated with the risk of NSCLC.

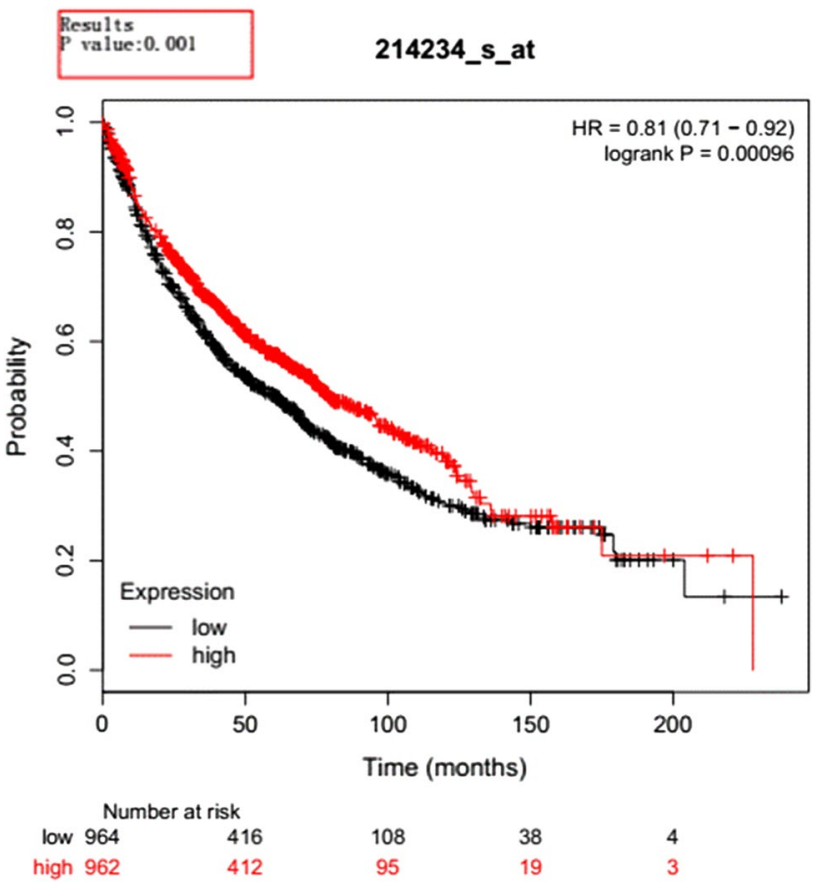

Fig. 2 Relationship between CYP3A5 gene and prognosis of lung squamous cell carcinoma. The $Y$-axis is survival rate. Red line represents low expression of CYP3A5 gene, and black line represents high expression of CYP3A5 gene in lung squamous carcinoma people; $P$ value $<0.05$ was considered statistically significant; $H R$ Hazard ratio; the numbers at the bottom indicate the number of people still alive at the different survival time (color figure online)

Acknowledgements We are grateful to the patients and control subjects for their participation in this study. We also thank the clinicians and hospital staff who contributed to the sample and data collection for this study.

Funding This research project was supported by Shaanxi Cooperation Project (2019KW-034).

\section{Compliance with ethical standards}

Conflict of interest The authors declare that there is no competing interest between them and this research project.

Availability of data and materials The data analyzed are available from the corresponding author on reasonable request. The datasets supporting the conclusions of this article are included within the article.

Ethical approval The purpose of this study was to investigate the relationship between CYP450 gene polymorphism and lung cancer susceptibility. This study was conducted in vitro and has no adverse effects on patients' health, because the samples used were the remaining samples after clinical examination and experimenters did not have direct contact with patients, so we applied for exemption from informed consent and 
got support from the Ethical Committees of the First Affiliated Hospital of Xi'an Jiaotong University (Acceptance No. KYLLSL-2018-265).

Informed consent This study only collected patients' gender, age, and clinical diagnosis data and did not involve patients' personal privacy. It did not provide test results to patients, and the privacy and identity information of subjects were guaranteed. This study is only a case-control study, not a basis for clinical diagnosis

Open Access This article is licensed under a Creative Commons Attribution 4.0 International License, which permits use, sharing, adaptation, distribution and reproduction in any medium or format, as long as you give appropriate credit to the original author(s) and the source, provide a link to the Creative Commons licence, and indicate if changes were made. The images or other third party material in this article are included in the article's Creative Commons licence, unless indicated otherwise in a credit line to the material. If material is not included in the article's Creative Commons licence and your intended use is not permitted by statutory regulation or exceeds the permitted use, you will need to obtain permission directly from the copyright holder. To view a copy of this licence, visit http://creativecommons.org/licenses/by/4.0/.

\section{References}

1. Jiang LP, Zhu ZT, He CY. Effects of CYP3A5 genetic polymorphism and smoking on the prognosis of non-small-cell lung cancer. Onco Targets Ther. 2016;9:1461-9.

2. Bai L, Shin S, Burnett RT, et al. Exposure to ambient air pollution and the incidence of lung cancer and breast cancer in the Ontario Population Health and Environment Cohort. Int J Cancer. 2020;146(9):2450-9.

3. Klupczynska A, Plewa S, Kasprzyk M, et al. Serum lipidome screening in patients with stage I non-small cell lung cancer. Clin Exp Med. 2019;19(4):505-13.

4. Torre LA, Bray F, Siegel RL, et al. Global cancer statistics, 2012. CA Cancer J Clin. 2015;65(2):87-108.

5. Sawrycki P, Domagalski K, Cechowska M, et al. Relationship between CYP1B1 polymorphisms (c.142C > G, c.355G $>$ T, c. $1294 \mathrm{C}>\mathrm{G}$ ) and lung cancer risk in Polish smokers. Future Oncol (London, England). 2018;14(16):1569-77.

6. Romaszko-Wojtowicz A, Bucinski A, Doboszynska A. Impact of smoking on multiple primary cancers survival: a retrospective analysis. Clin Exp Med. 2018;18(3):391-7.

7. Liang Y, Thakur A, Gao L, et al. Correlation of CLPTM1L polymorphisms with lung cancer susceptibility and response to cisplatin-based chemotherapy in a Chinese Han population. Tumour Biol. 2014;35(12):12075-82.

8. Nicos M, Krawczyk P, Jarosz B, et al. Analysis of KRAS and BRAF genes mutation in the central nervous system metastases of non-small cell lung cancer. Clin Exp Med. 2016;16(2):169-76.

9. Roviello G, Zanotti L, Cappelletti MR, et al. Are EGFR tyrosine kinase inhibitors effective in elderly patients with EGFR-mutated non-small cell lung cancer? Clin Exp Med. 2018;18(1):15-20.

10. Eun HS, Cho SY, Lee BS, Seong IO, Kim KH. Profiling cytochrome P450 family 4 gene expression in human hepatocellular carcinoma. Mol Med Rep. 2018;18(6):4865-76.

11. Hashimoto H, Nakagawa T, Yokoi T, et al. Fetus-specific CYP3A7 and adult-specific CYP3A4 expressed in Chinese hamster CHL cells have similar capacity to activate carcinogenic mycotoxins. Can Res. 1995;55(4):787-91.

12. Gervasini G, Garcia-Martin E, Ladero JM, et al. Genetic variability in CYP3A4 and CYP3A5 in primary liver, gastric and colorectal cancer patients. BMC Cancer. 2007;7:118.
13. Yu W, Chen L, Yang YQ, et al. Cytochrome P450 omega-hydroxylase promotes angiogenesis and metastasis by upregulation of VEGF and MMP-9 in non-small cell lung cancer. Cancer Chemother Pharmacol. 2011;68(3):619-29.

14. Yeh KT, Chen JC, Chen CM, et al. CYP3A5*1 is an inhibitory factor for lung cancer in Taiwanese. Kaohsiung J Med Sci. 2003;19(5):201-7.

15. Alexanian A, Miller B, Roman RJ, Sorokin A. 20-HETE-producing enzymes are up-regulated in human cancers. Cancer Genom Proteom. 2012;9(4):163-9.

16. Gandhi AV, Saxena S, Relles D, et al. Differential expression of cytochrome P450 omega-hydroxylase isoforms and their association with clinicopathological features in pancreatic ductal adenocarcinoma. Ann Surg Oncol. 2013;20(Suppl 3):S636-43.

17. Colombero C, Papademetrio D, Sacca P, et al. Role of 20-hydroxyeicosatetraenoic acid (20-HETE) in androgen-mediated cell viability in prostate cancer cells. Horm Cancer. 2017;8(4):243-56.

18. Kim WY, Lee SJ, Min J, et al. Identification of novel CYP4F2 genetic variants exhibiting decreased catalytic activity in the conversion of arachidonic acid to 20-hydroxyeicosatetraenoic acid (20-HETE). Prostaglandins Leukot Essent Fatty Acids. 2018;131:6-13.

19. Stec DE, Roman RJ, Flasch A, Rieder MJ. Functional polymorphism in human CYP4F2 decreases 20-HETE production. Physiol Genom. 2007;30(1):74-81.

20. Johnson AL, Edson KZ, Totah RA, Rettie AE. Cytochrome P450 omega-hydroxylases in inflammation and cancer. Adv Pharmacol. 2015;74:223-62.

21. Shankar A, Borin TF, Iskander A, et al. Combination of vatalanib and a 20-HETE synthesis inhibitor results in decreased tumor growth in an animal model of human glioma. Onco Targets Ther. 2016;9:1205-19.

22. Dandara C, Ballo R, Parker MI. CYP3A5 genotypes and risk of oesophageal cancer in two South African populations. Cancer Lett. 2005;225(2):275-82.

23. He XF, Liu ZZ, Xie JJ, et al. Association between the CYP3A4 and CYP3A5 polymorphisms and cancer risk: a meta-analysis and meta-regression. Tumour Biol. 2014;35(10):9859-77.

24. Wang BS, Liu Z, Xu WX, Sun SL. CYP3A5*3 polymorphism and cancer risk: a meta-analysis and meta-regression. Tumour Biol. 2013;34(4):2357-66.

25. Yu T, Wang X, Zhu G, et al. The prognostic value of differentially expressed CYP3A subfamily members for hepatocellular carcinoma. Cancer Manag Res. 2018;10:1713-26.

26. Islam MS, Mostofa AG, Ahmed MU, et al. Association of CYP3A4, CYP3A5 polymorphisms with lung cancer risk in Bangladeshi population. Tumour Biol. 2014;35(2):1671-8.

27. Jiang F, Chen L, Yang YC, et al. CYP3A5 functions as a tumor suppressor in hepatocellular carcinoma by regulating mTORC2/ Akt signaling. Cancer Res. 2015;75(7):1470-81.

28. Lamba JK, Lin YS, Schuetz EG, Thummel KE. Genetic contribution to variable human CYP3A-mediated metabolism. Adv Drug Deliv Rev. 2002;54(10):1271-94.

29. Diekstra MH, Swen JJ, Boven E, et al. CYP3A5 and ABCB1 polymorphisms as predictors for sunitinib outcome in metastatic renal cell carcinoma. Eur Urol. 2015;68(4):621-9.

30. Sim S, Bergh J, Hellstrom M, Hatschek T, Xie H. Pharmacogenetic impact of docetaxel on neoadjuvant treatment of breast cancer patients. Pharmacogenomics. 2018;19(16):1259-68.

Publisher's Note Springer Nature remains neutral with regard to jurisdictional claims in published maps and institutional affiliations. 